ISSN 1980-5098 (1) @ DOI: http://dx.doi.org/10.5902/1980509831603

\title{
FUNGOS MICORRÍZICOS ARBUSCULARES E SERAPILHEIRA COMO INDICADORES DO EFEITO DE BORDA EM FRAGMENTO DE FLORESTA ESTACIONAL
}

\author{
MYCORRHIZAL FUNGI AND LITTER AS INDICATORS OF THE EDGE EFFECT IN A \\ FRAGMENT SEASONAL FOREST
}

Renata Soares dos Santos ${ }^{1}$ Patricia Anjos Bittencourt Barreto-Garcia ${ }^{2}$ Rafael Nogueira Scoriza ${ }^{3}$

\section{RESUMO}

As atividades humanas causadoras de desmatamentos favorecem a fragmentação e a formação de bordas na floresta, modificando as características bióticas e abióticas do ecossistema, o que pode ser avaliado através de indicadores do solo. Com isso, o objetivo deste estudo foi avaliar o estoque de serapilheira e a comunidade de fungos micorrízicos arbusculares (FMA) como indicadores do efeito de borda em um fragmento de Floresta Estacional em Vitória da Conquista, Bahia. Foram realizadas amostragens ao final de cada estação do ano. Para isso, foram delimitadas quatro faixas na direção borda-matriz (borda - 0-10 $\mathrm{m}$, faixa $1-40-50 \mathrm{~m}$, faixa $2-80-90 \mathrm{~m}$ e interior $-400 \mathrm{~m}$ ). A serapilheira acumulada (quatro repetições por faixa) foi coletada com o auxílio de um gabarito de madeira (área $0,25 \mathrm{~m}^{2}$ ). Nos mesmos pontos em que se coletou a serapilheira foram coletadas amostras de solo (camada 0-5 cm) para extração dos esporos de fungos micorrízicos. O efeito de borda em fragmentos de Floresta Estacional Semidecidual pode alterar o estoque de serapilheira com redução de massa nos locais mais próximos da borda. A comunidade de fungos micorrízicos arbusculares apresentou a ocorrência de espécies exclusivas em determinadas faixas no gradiente borda-interior do fragmento.

Palavras-chave: fragmentação; fitomassa acumulada; sazonalidade; comunidade de FMA.

\begin{abstract}
Deforestation causes the formation of forest fragments and edges, changing the biotic and abiotic conditions of a forest ecosystem. The edge effects in a forest can be assessed by soil indicators. The objective of this study was to evaluate the litter stock and arbuscular mycorrhizal fungal community as edge effect indicators in a fragment of a seasonal forest in Vitória da Conquista, Bahia state. Sampling was carried out at the end of each season. Four sampling ranges were established in the edge-interior direction (edge: $0-10 \mathrm{~m}$, range 1: $40-50 \mathrm{~m}$, range 2: $80-90 \mathrm{~m}$, and interior: $400 \mathrm{~m}$ ). The accumulated litter was collected four times using a wooden template (area $0.25 \mathrm{~m}^{2}$ ). The soil samples (layer $0-5 \mathrm{~cm}$ ) for extracting the spores of mycorrhizal fungi were collected from the same litter collection areas. The edge effect in areas of a seasonal forest can change the litter stock, showing a mass reduction closer to the edge. The community of mycorrhizal fungi shows the occurrence of unique species in the gradient of edge-interior fragment.
\end{abstract}

Keywords: fragmentation; biomass accumulated; seasonality; community AMF.

\section{INTRODUÇÃO}

Da área total original de Floresta Atlântica, estima-se que apenas 11,7\% esteja preservada em

1 Engenheira Florestal, MSc., Doutoranda em Agronomia, Universidade Estadual do Norte Fluminense Darcy Ribeiro, Av. Alberto Lamego, 2000, Horto, CEP 28013-602, Campos dos Goytacazes (RJ), Brasil. renata2soares@, yahoo.com.br

2 Engenheira Florestal, Dra ${ }^{\mathrm{a}}$, Professora Adjunta do Departamento de Engenharia Agrícola e Solos, Universidade Estadual do Sudoeste da Bahia, Estrada do Bem Querer, km 4, Cidade Universitária, CEP 45083-900, Vitória da Conquista (BA), Brasil. patriciabarreto@uesb.edu.br

3 Biólogo, MSc., Doutorando em Agronomia, Universidade Federal Rural do Rio de Janeiro, Rodovia BR 465, km 7, Zona Rural, CEP 23890-000, Seropédica (RJ), Brasil. rafaelscoriza@gmail.com

Recebido para publicação em 13/03/2014 e aceito em 4/10/2016

Ci. Fl., v. 28, n. 1, jan.- mar., 2018 
remanescentes florestais, especialmente da formação estacional em estádio secundário da sucessão natural, o que corresponde a uma área de 15.719.337 ha (RIBEIRO et al., 2009; BRASIL, 2010). A situação da Mata Atlântica do estado da Bahia não difere da observada em outros lugares do país, de uma área original de $36 \%$ do território do estado restam menos de 6\% (NÚCLEO MATA ATLÂNTICA, 2013). Os fragmentos de mata decídua florestal, conhecidos regionalmente como mata de cipó, estão sob forte pressão do avanço de atividades agropastoris, em especial com a cultura do café na região que abrange os municípios de Vitória da Conquista, Jequié e Boa Nova (BATISTA; TIMMERS; CUNHA, 2006). A análise desses ecossistemas deve ser o ponto de partida para o desenvolvimento de estratégias de conservação, uma vez que requerem ações de manejo e proteção contra perturbações antrópicas.

A fragmentação dos ecossistemas florestais afeta, além da diversidade e a inter-relação entre as espécies, muitas das suas funções e características, como a biomassa florestal e os ciclos bioquímico, hidrológico e do carbono (LAURANCE et al., 2011). Além disso, por provocar a divisão de uma população existente em duas ou mais, aumenta as quantidades de margens e de bordas abruptas, que ficam expostas à maior insolação, exposição ao vento e dessecamento (LAURANCE et al., 2003). Este processo tem sido cada mais frequente, ocorrendo nas mais diversas condições do mundo, como consequência das atividades humanas voltadas para a construção de moradias, estabelecimento de áreas agrícolas e para a extração de recursos florestais (HARPER et al., 2005).

A borda consiste na zona de contato entre um ambiente conservado e outro antropizado (SCARIOT et al., 2003), o que promove a criação de um gradiente e a influência de um ambiente sobre o outro, originando diversas alterações estruturais e funcionais no ecossistema. Estes efeitos de borda atingem centenas de metros na região interna da floresta (HARPER et al., 2005; LAURANCE et al., 2011), embora sejam mais frequentes nos primeiros 30 metros (RIGUEIRA et al., 2012).

As alterações resultantes dos efeitos de borda podem ser avaliadas através de indicadores ambientais, que permitem identificar o estado de conservação de fragmentos florestais. Esses indicadores constituem-se da agregação de diferentes e múltiplos dados, que representam um fenômeno em estudo (EUROPEAN ENVIRONMENT AGENCY, 2003). O uso de informações acerca de propriedades ou processos biológicos do solo, por exemplo, representa uma importante ferramenta para avaliar e interpretar os efeitos de interferências naturais ou antrópicas no solo, especialmente porque este compartimento do ecossistema é produto da atuação do clima e dos organismos sobre o material de origem ao longo do tempo, sendo, portanto, considerado uma variável integradora (FERREIRA JÚNIOR; SCHAEFER; SILVA, 2012; HEGER; IMFELD; MITCHELL, 2012). Como o solo é composto por propriedades físicas, químicas e biológicas, indicadores que relacionem todos estes fatores apresentam grande potencial de responder a alterações impostas no meio, sendo considerados relevantes para uma completa descrição da qualidade do solo (STENBERG, 1999).

Dentre as consequências das perturbações ocasionadas pelo efeito de borda surge a alteração da dinâmica da serapilheira, ocasionado principalmente pelo aumento da queda de folhas e ramos, além da intensificação da mortalidade de árvores (LAURANCE et al., 1998; 2002; FAHRIG, 2003), principalmente as tolerantes à sombra. Isto ocasiona a predominância de espécies pioneiras de crescimento rápido (PRIMACK; RODRIGUES, 2001), que possuem alta taxa de renovação e, com isso, promovem grande aporte de fitomassa nas bordas (BROWN; LUGO, 1990; GASCON; WILLIAMSON; FONSECA, 2000). Além de ser influenciada pela composição de espécies e estádio sucessional da floresta, a fitomassa depositada também tem efeito de diversos fatores como o clima e a fertilidade do solo (VITOUSEK; SANFORD JÚNIOR, 1986). Por essa razão, a utilização da serapilheira no monitoramento de áreas florestais é válida pelo seu potencial como "indicador de reação", capaz de responder às modificações do ambiente (MACHADO; PIÑA-RODRIGUES; PEREIRA, 2008).

As comunidades de microrganismos do solo também são sensíveis às alterações do meio e ao estresse ambiental, característica que permite sua utilização como indicadores biológicos do solo (PAGANO et al., 2011; HEGER; IMFELD; MITCHELL, 2012). Exemplo disso são os fungos micorrízicos arbusculares (FMA), que estão presentes nos solos dos mais diversos ecossistemas, formando a associação simbiótica denominada micorriza arbuscular (SMITH; READ, 2008). Esses organismos contribuem para a estruturação do solo e retenção de água, por meio da formação de micro e macroagregados (PENG; GUO; LIU, 2013) e incrementam a dinâmica e aporte de nutrientes ao solo via serapilheira, em razão do aumento 
de produtividade de biomassa vegetal que proporcionam (LEAKE et al., 2004).

Além disso, os FMA são influenciados pelo grau de suscetibilidade das plantas à simbiose, pelo manejo do solo e pela fragmentação florestal (PAGANO et al., 2011; GRILLI; URCELAY; GALETTO, 2012). Desse modo, a avaliação de comunidades nativas desses organismos pode permitir inferir mais eficientemente sobre a sua diversidade funcional, avaliar o padrão de sucessão de uma comunidade vegetal e servir como instrumento de medida da qualidade do solo (STÜRMER; SIQUEIRA, 2010).

Com isso, o objetivo deste estudo foi avaliar o efeito de borda em um fragmento de Floresta Estacional Semidecidual Montana em Vitória da Conquista - BA, utilizando como indicadores o estoque de serapilheira florestal e a comunidade de fungos micorrízicos arbusculares do solo.

\section{MATERIAL E MÉTODOS}

\section{Caracterização da área}

O estudo foi realizado em um fragmento florestal localizado na Universidade Estadual do Sudoeste da Bahia (UESB), em Vitória da Conquista - BA. O fragmento, que se encontra em estágio médio de regeneração (CONAMA no 1 de 1994), ocupa uma área de 42 ha e está situado a $891 \mathrm{~m}$ de altitude, nas coordenadas $14^{\circ} 52^{\prime} 46^{\prime \prime} \mathrm{S}$ e $40^{\circ} 47^{\prime} 34^{\prime}$ 'W. A vegetação dominante é classificada como Floresta Estacional Semidecidual Montana (IBGE, 2012), conhecida regionalmente como mata de cipó. Trata-se de uma floresta relativamente baixa (árvores com altura entre 10 e $15 \mathrm{~m}$ ), composta de mesofanerófitos parcialmente caducifólios, envolvidos por lianas, com predominância de ecótipos da família Leguminosae, destacandose o gênero Parapiptadenia. A área do entorno do fragmento florestal é constituída predominantemente de pastagem nativa degradada, utilizada para bovinos. Uma estrada de cerca de dois metros de largura circunda maior parte do perímetro do fragmento, separando-o da área de pastagem.

De acordo com a classificação de Köppen, o clima da região é tropical de altitude (Cwb) (ALVARES et al., 2013). Os valores médios de temperatura e precipitação no período de estudo (setembro de 2011 a agosto de 2012) estão apresentados na Figura 1. O solo é classificado como Latossolo Amarelo distrófico, segundo EMBRAPA (2013).

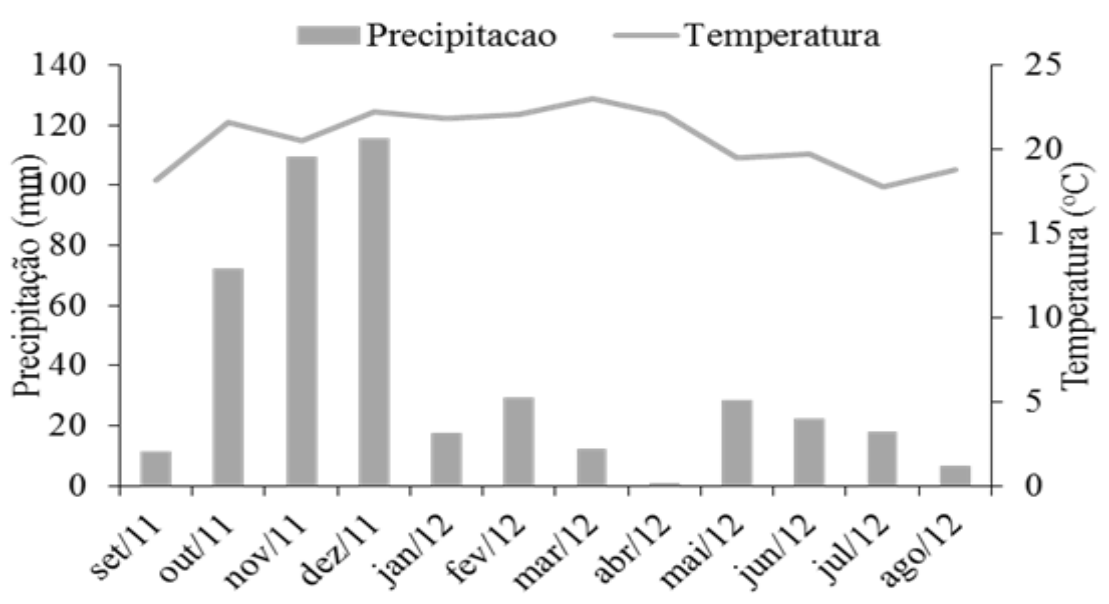

FIGURA 1: Precipitação e temperatura média do período do estudo em Vitória da Conquista, Bahia. Dados fornecidos pela Estação Meteorológica da Universidade Estadual do Sudoeste da Bahia (ESMET).

FIGURE 1: Precipitation and average temperature of the study periods in Vitória da Conquista, Bahia state. Data provided by the Meteorological Station of the State University of Southwest Bahia (ESMET).

\section{Delimitação das faixas}

Para avaliar o efeito de borda, foram delimitadas quatro faixas de acordo com a distância da borda: (1) borda - posicionada na extremidade da mata, a 0-10 m da borda; (2) faixa 1 - alocada a 40-50 m da 
borda, representando uma primeira zona de transição dentro da mata; (3) faixa 2 - posicionada a 80-90 m da borda, representando uma segunda zona intermediária dentro da mata e (4) interior - correspondendo à matriz do fragmento, localizada a cerca de $400 \mathrm{~m}$ de distância da borda (metade da distância total entre extremidades do fragmento).

\section{Coleta de serapilheira e de solo}

As coletas foram realizadas por um ano, trimestralmente, totalizando quatro coletas, nos meses de novembro de 2011 e fevereiro, maio e agosto de 2012, que correspondem ao período final de cada uma das estações do ano (primavera, verão, outono e inverno, respectivamente).

A serapilheira acumulada foi amostrada com quatro repetições aleatórias em cada faixa, utilizando uma moldura de madeira $0,25 \mathrm{~m}^{2}(0,5 \times 0,5 \mathrm{~m})$. Após coletada, a serrapilheira foi triada nas frações folhas, galhos, cascas, estruturas reprodutivas e material amorfo. Posteriormente, o material foi seco em estufa a $60^{\circ} \mathrm{C}$ por 72 horas e pesados.

A comunidade de FMA do solo foi amostrada com quatro repetições na profundidade de $0-5 \mathrm{~cm}$, no solo abaixo da serapilheira coletada. A extração dos esporos seguiu a técnica adaptada de peneiramento úmido (GERDEMANN; NICOLSON, 1963) e centrifugação em gradiente de densidade (JENKINS, 1964). Os esporos foram contabilizados e separados em dois grupos em uma lâmina de microscopia (um com álcool polivinil lactoglicerol (PVLG) e o outro fixado com PVLG acrescido do reagente Melzer). A identificação das espécies foi realizada por comparação a partir dos trabalhos de Schenck e Perez (1987), do International Culture Collection of (Vesicular) Arbuscular Mycorrhizal Fungi - INVAM (http://invam.caf.wvu.edu/) e do International Mycologycal Association (http://www.mycobank.org).

\section{Análise dos Dados}

Os valores do estoque de serrapilheira foram convertidos para $\mathrm{Mg} \mathrm{ha}^{-1}$. O número de esporos foi representado em densidade de esporos $\mathrm{g}^{-1}$ de solo. A diversidade alfa da comunidade de FMA foi calculada por meio do índice Margalef $(\alpha)$ através da equação $\alpha=(\mathrm{S}-1) \cdot(\log \mathrm{N})^{-1}$, em que s é o número de espécies amostradas e $\mathrm{N}$ é o número total de indivíduos (esporos) em todas as espécies. Os dados foram testados quanto à homogeneidade (teste de Cochran) e normalidade (teste de Lilliefors), por meio do programa estatístico $S A E G{ }^{\circledR}$ v.9.1. A comparação de médias foi realizada por meio da análise de variância, segundo o delineamento inteiramente casualizado com quatro tratamentos e quatro repetições, com aplicação do teste de Tukey, a $5 \%$ com o software SISVAR ${ }^{\circledR}$ v.5.3. A relação entre as variáveis climáticas e os indicadores foram avaliadas pelas correlações de Pearson a 5\%, utilizando-se o programa SAEG® v.9.1.

\section{RESULTADOS E DISCUSSÃO}

\section{Estoque de serapilheira}

O estoque médio de serapilheira no fragmento florestal foi de $8,2 \pm 0,5 \mathrm{Mg} \mathrm{ha}^{-1}$, superior ao verificado em fragmentos florestais semelhantes em outros estudos (GARCIA et al., 2005; KLEINPAUL et al., 2005; SPERANDIO et al., 2012). Este resultado pode estar associado a uma menor taxa de decomposição (NUNES \& PINTO, 2012) ou a uma predominância de espécies pioneiras capazes de promover grande aporte de fitomassa (BROWN; LUGO, 1990; GASCON et al., 2000; SOARES FILHO, 2012).

A fração galhos representou $32 \%$ e, quando somada com a fração cascas, $38 \%$ do estoque total de serapilheira total. Barbosa et al. (2012), estudando uma Floresta Estacional Semidecidual na mesma região deste estudo, verificaram uma representatividade semelhante da fração folhas (40\%), atribuindo o resultado a uma elevada taxa de decomposição do material foliar, considerando a grande ocorrência de espécies das famílias Leguminosae e Fabaceae no fragmento.

A fração galhos representou $32 \%$ e quando somada com a fração cascas $38 \%$ do estoque total de serapilheira. A grande participação destas frações deve estar relacionada com composição química e orgânica desses materiais, que provoca menor acessibilidade física a compostos carbônicos pelos organismos 
decompositores (VIEIRA et al., 2010; NUNES; PINTO, 2012). A fração material amorfo contribuiu com $23 \%$ e a fração material reprodutivo com apenas $1 \%$.

Ao se analisar a proporção das frações no gradiente borda-matriz do fragmento, verificou-se que os galhos foram os componentes predominantes da serapilheira na borda, representando $40 \%$ do estoque total, enquanto a fração folhas teve maior participação nas demais faixas $(33,43$ e 42 \% nas faixas 1,2 e interior, respectivamente). Isto evidencia a ocorrência de um efeito de borda mais intenso na faixa mais externa, possivelmente em decorrência da maior exposição a ventos e chuvas, que provocou maior desprendimento de galhos.

A serapilheira total acumulada foi superior no interior do fragmento em relação às demais faixas. Esta mesma tendência também foi verificada para as frações folhas e material amorfo. No entanto, ao analisar o acúmulo nas diferentes estações observaram-se respostas diferenciadas (Tabela 1).

TABELA 1: Estoque das frações e do total de serapilheira acumulada $\left(\mathrm{Mg} \mathrm{ha}^{-1}\right)$ nas faixas do fragmento florestal nas diferentes estações do ano.

TABLE 1: Accumulated litter and fractions $\left(\mathrm{Mg} \mathrm{ha}^{-1}\right)$ in the forest fragment tracks in different seasons.

\begin{tabular}{|c|c|c|c|c|c|c|}
\hline Tratamento & Folhas & Galhos & Cascas & $\begin{array}{l}\text { Material } \\
\text { reprodutivo }\end{array}$ & $\begin{array}{l}\text { Material } \\
\text { amorfo }\end{array}$ & Total \\
\hline & \multicolumn{6}{|c|}{ Média estações } \\
\hline Borda & $2,63 \mathrm{~b}$ & $3,06 \mathrm{a}$ & $0,46 \mathrm{a}$ & $0,04 \mathrm{a}$ & $1,52 \mathrm{~b}$ & $7,72 \mathrm{~b}$ \\
\hline Faixa 1 & $2,22 \mathrm{~b}$ & $1,95 \mathrm{a}$ & $0,56 \mathrm{a}$ & $0,05 \mathrm{a}$ & $1,97 \mathrm{ab}$ & $6,75 \mathrm{~b}$ \\
\hline Faixa 2 & $2,87 \mathrm{~b}$ & $2,13 \mathrm{a}$ & $0,26 \mathrm{a}$ & $0,07 \mathrm{a}$ & $1,16 \mathrm{~b}$ & $6,67 \mathrm{~b}$ \\
\hline \multirow[t]{2}{*}{ Interior } & $4,30 \mathrm{a}$ & $2,70 \mathrm{a}$ & $0,43 \mathrm{a}$ & $0,22 \mathrm{a}$ & $2,67 \mathrm{a}$ & $10,32 \mathrm{a}$ \\
\hline & \multicolumn{6}{|c|}{ Primavera } \\
\hline Borda & $3,30 \mathrm{Aa}$ & $2,62 \mathrm{aA}$ & $0,41 \mathrm{aA}$ & $0,03 \mathrm{aA}$ & $0,15 \mathrm{aB}$ & $6,52 \mathrm{Aa}$ \\
\hline Faixa 1 & $2,63 \mathrm{aA}$ & $1,56 \mathrm{aAB}$ & $0,69 \mathrm{aA}$ & $0,08 \mathrm{aA}$ & $1,09 \mathrm{aB}$ & $6,05 \mathrm{Aa}$ \\
\hline Faixa 2 & $3,25 \mathrm{aA}$ & $1,72 \mathrm{aA}$ & $0,35 \mathrm{aA}$ & $0,06 \mathrm{aA}$ & $0,67 \mathrm{aC}$ & $6,05 \mathrm{aA}$ \\
\hline \multirow[t]{2}{*}{ Interior } & $4,34 \mathrm{aAB}$ & $1,60 \mathrm{aA}$ & $0,31 \mathrm{aA}$ & $0,08 \mathrm{aA}$ & $1,27 \mathrm{aB}$ & $7,60 \mathrm{Aa}$ \\
\hline & \multicolumn{6}{|c|}{ Verão } \\
\hline Borda & $2,61 \mathrm{aA}$ & $2,86 \mathrm{aA}$ & $0,65 \mathrm{aA}$ & $0,05 \mathrm{aA}$ & $1,52 \mathrm{abAB}$ & $7,69 \mathrm{abA}$ \\
\hline Faixa 1 & $1,86 \mathrm{aA}$ & $1,14 \mathrm{aB}$ & $0,44 \mathrm{aA}$ & $0,08 \mathrm{aA}$ & $2,44 \mathrm{aAB}$ & $5,95 \mathrm{bA}$ \\
\hline Faixa 2 & $1,87 \mathrm{aA}$ & $3,03 \mathrm{aA}$ & $0,14 \mathrm{Aa}$ & $0,14 \mathrm{aA}$ & $0,87 \mathrm{bBC}$ & $6,05 \mathrm{bA}$ \\
\hline \multirow[t]{2}{*}{ Interior } & $3,15 \mathrm{aB}$ & $3,63 \mathrm{aA}$ & $0,21 \mathrm{Aa}$ & $0,63 \mathrm{aA}$ & $2,37 \mathrm{aAB}$ & $9,98 \mathrm{aA}$ \\
\hline & \multicolumn{6}{|c|}{ Outono } \\
\hline Borda & $2,39 \mathrm{cA}$ & $2,61 \mathrm{aA}$ & $0,20 \mathrm{aA}$ & $0,04 \mathrm{aA}$ & $2,16 \mathrm{bA}$ & $7,41 \mathrm{bA}$ \\
\hline Faixa 1 & $2,03 \mathrm{cA}$ & $2,29 \mathrm{aAB}$ & $0,34 \mathrm{aA}$ & $0,03 \mathrm{aA}$ & $1,39 \mathrm{bAB}$ & $6,10 \mathrm{bA}$ \\
\hline Faixa 2 & $3,65 \mathrm{bA}$ & $4,03 \mathrm{aA}$ & $0,95 \mathrm{aA}$ & $0,06 \mathrm{aA}$ & $2,51 \mathrm{bA}$ & $11,2 \mathrm{abA}$ \\
\hline \multirow[t]{2}{*}{ Interior } & $5,25 \mathrm{aA}$ & $3,14 \mathrm{aA}$ & $0,98 \mathrm{aA}$ & $0,09 \mathrm{aA}$ & $3,45 \mathrm{aA}$ & $12,91 \mathrm{aA}$ \\
\hline & \multicolumn{6}{|c|}{ Inverno } \\
\hline Borda & $2,22 \mathrm{bA}$ & $4,15 \mathrm{aA}$ & $0,58 \mathrm{aA}$ & $0,05 \mathrm{aA}$ & $2,26 \mathrm{aA}$ & $9,26 \mathrm{aA}$ \\
\hline Faixa 1 & $2,34 \mathrm{bA}$ & $2,80 \mathrm{aA}$ & $0,78 \mathrm{aA}$ & $0,02 \mathrm{aA}$ & $2,97 \mathrm{aA}$ & $8,91 \mathrm{aA}$ \\
\hline Faixa 2 & $3,49 \mathrm{abA}$ & $2,27 \mathrm{aA}$ & $0,50 \mathrm{aA}$ & $0,05 \mathrm{aA}$ & $2,04 \mathrm{aAB}$ & $8,35 \mathrm{aA}$ \\
\hline Interior & $4,47 \mathrm{aAB}$ & $2,44 \mathrm{aA}$ & $0,22 \mathrm{aA}$ & $0,07 \mathrm{aA}$ & $3,60 \mathrm{aA}$ & $10,79 \mathrm{aA}$ \\
\hline
\end{tabular}

Em que: valores seguidos de letras minúsculas iguais, na coluna, que comparam o estoque de serapilheira entre as faixas; e as letras maiúsculas iguais, que comparam o estoque de serapilheira de cada uma das faixas entre as estações não diferem entre si pelo teste de Tukey a 5\% de significância.

Na primavera não foram verificadas diferenças entre as faixas. No verão, o total de serapilheira estocada apresentou maior valor no interior do fragmento em relação às faixas 1 e 2. Para esta estação, apenas a fração material amorfo apresentou diferenças entre faixas, mas com pequena variação. No outono 
e no inverno, as diferenças entre a borda e a matriz do fragmento foram mais marcantes, principalmente para as frações folhas e material amorfo, que apresentaram maior estoque na matriz em relação à borda e faixas 1 e 2 (Tabela 1). Ainda no outono, a serapilheira total também apresentou maiores valores no interior, em comparação à borda e faixa 1 (Tabela 1). Dentre os efeitos sobre a serapilheira, espera-se um aumento na queda de folhas e uma menor taxa de decomposição na região da borda do fragmento, o que gera maior acúmulo (LAURANCE et al. 1998; 2002). Entretanto, ocorreu o oposto neste estudo, o que pode estar associado à maior densidade, número de espécies e biomassa florestal na matriz, conferindo maior valor de deposição e estoque de serapilheira no solo (RIGUEIRA et al., 2012; DINIZ et al., 2015), o que também foi observado por Vogel et al. (2013).

Comparando-se os estoques de serapilheira de cada uma das faixas entre as estações, apenas na faixa 2 e no interior foram observadas variações das frações folhas e material amorfo, que apresentaram maiores acúmulos no outono e no inverno (Tabela 1). Nestas mesmas estações foi observada maior diferenciação destas frações entre borda e interior do fragmento, conforme mencionado anteriormente.

$\mathrm{Na}$ região de estudo ocorreu um período de temperaturas mais amenas entre os meses de maio e agosto (Figura 1), constatando-se correlações negativas entre esta variável climática e os estoques de serapilheira das faixas 1 e 2 e da matriz do fragmento (Tabela 2). Tal resultado demonstra uma redução do acúmulo de fitomassa nos períodos mais quentes, possivelmente, em decorrência de um aumento da taxa de decomposição.

TABELA 2: Coeficiente de correlação de Pearson do estoque de serapilheira nas faixas do fragmento florestal com as variáveis climáticas temperatura e precipitação.

TABLE 2: Pearson correlation coefficient of the accumulated litter in the bands of forest fragment climate variables of temperature and precipitation.

\begin{tabular}{ccccccc}
\hline Tratamento & Folhas & Galhos & Cascas & $\begin{array}{c}\text { Material } \\
\text { reprodutivo }\end{array}$ & $\begin{array}{c}\text { Material } \\
\text { amorfo }\end{array}$ & Total \\
\hline & Temperatura & & & & & \\
Borda & 0,45 & $-0,54$ & 0,41 & $-0,19$ & $-0,47$ & $-0,48$ \\
Faixa 1 & $-0,39$ & $\mathbf{- 0 , 9 7}$ & $-0,38$ & $\mathbf{0 , 8 9}$ & $-0,10$ & $-0,69$ \\
Faixa 2 & $\mathbf{- 0 , 7 7}$ & $-0,02$ & $-0,72$ & $\mathbf{0 , 9 1}$ & $\mathbf{- 0 , 7 9}$ & $\mathbf{- 0 , 8 1}$ \\
Interior & $\mathbf{- 0 , 8 3}$ & 0,38 & $-0,36$ & $\mathbf{0 , 8 7}$ & $-0,62$ & $-0,43$ \\
\hline & Precipitação & & & & & \\
Borda & $\mathbf{0 , 9 8}$ & $-0,60$ & $-0,24$ & $\mathbf{- 0 , 9 4}$ & $\mathbf{- 0 , 9 7}$ & $\mathbf{- 0 , 8 4}$ \\
Faixa 1 & 0,66 & $-0,50$ & 0,19 & 0,69 & $\mathbf{- 0 , 7 8}$ & $-0,54$ \\
Faixa 2 & 0,16 & $-0,54$ & $-0,26$ & $-0,13$ & $-0,73$ & $-0,39$ \\
Interior & $-0,01$ & $-0,69$ & $-0,11$ & $-0,29$ & $\mathbf{- 0 , 9 1}$ & $\mathbf{- 0 , 7 8}$ \\
\hline
\end{tabular}

Em que: números destacados em negrito correspondem a correlações significativas a $10 \%$ de probabilidade de erro.

Por outro lado, para a precipitação foram verificadas associações significativas nas faixas mais próximas à borda, embora também tenha ocorrido na matriz (Tabela 2). Na borda, o acúmulo de folhas associou-se positivamente com a precipitação, sugerindo maior deposição e acúmulo desta fração em períodos mais chuvosos. Ainda na borda, os acúmulos de material amorfo correlacionaram-se negativamente com a variação pluviométrica (Tabela 2), o que indica uma possível aceleração da decomposição em períodos com maior disponibilidade de água, especialmente porque esta fração é originada do processo de fragmentação e decomposição da serapilheira pelos organismos do solo. De acordo com Riutta et al. (2012), a interferência da fragmentação do habitat e mudança do microclima na decomposição ocorrem em função das alterações na abundância, diversidade, característica funcional e distribuição dos macrodecompositores, o que cria diferenças entre borda e matriz e normalmente propicia maior velocidade de decomposição no interior do fragmento.

Em outras palavras, com base nos resultados discutidos anteriormente, nota-se que no interior do fragmento o estoque de serapilheira foi mais influenciado pela temperatura, enquanto nas faixas mais próximas da borda foi a precipitação que influenciou de forma mais marcante. Tal fato evidencia a ocorrência 
do gradiente climático criado pelo efeito de borda e sua implicação sobre o estoque de serapilheira.

\section{Comunidade de FMA do solo}

A densidade de esporos de FMA não diferiu entre as faixas do fragmento florestal. Todavia, ao se analisarem os resultados das estações separadamente verificaram-se variações na primavera (novembro de 2011), período com maior precipitação (Figura 1), quando houve maior densidade de esporos na borda; e no inverno (agosto de 2012), época mais fria e seca do ano (Figura 1), quando ocorreu maior densidade no interior do fragmento (Tabela 3 ).

TABELA 3: Densidade de esporos (esporos $\mathrm{g}^{-1}$ solo), número total de espécies e Índice de Margalef encontrados na comunidade de FMA presente nas faixas do fragmento florestal.

TABLE 3: Spore densities (spores $\mathrm{g}^{-1}$ soil), total number of species, and Margalef indices of the arbuscular mycorrhizal fungal (AMF) communities present in the bands of the forest fragment.

\begin{tabular}{|c|c|c|c|}
\hline Tratamento & Esporos $\mathrm{g}^{-1}$ solo & Número de espécies & Índice Margalef \\
\hline & \multicolumn{3}{|c|}{ Média estações } \\
\hline Borda & $9,5 \pm 0,7 \mathrm{a}$ & 6,5 & 5,6 \\
\hline Faixa 1 & $9,0 \pm 0,6 \mathrm{a}$ & 6,0 & 5,2 \\
\hline Faixa 2 & $10,0 \pm 0,8 \mathrm{a}$ & 7,3 & 6,3 \\
\hline \multirow[t]{2}{*}{ Interior } & $11,0 \pm 1,4 \mathrm{a}$ & 6,3 & 5,0 \\
\hline & \multicolumn{3}{|c|}{ Primavera } \\
\hline Borda & $7,9 \pm 0,1 \mathrm{aA}$ & 8 & 7,8 \\
\hline Faixa 1 & $3,5 \pm 0,5 \mathrm{bC}$ & 4 & 5,5 \\
\hline Faixa 2 & $4,0 \pm 0,5 \mathrm{bC}$ & 6 & 8,2 \\
\hline \multirow[t]{2}{*}{ Interior } & $4,2 \pm 0,7 \mathrm{bB}$ & 8 & 11,2 \\
\hline & \multicolumn{3}{|c|}{ Verão } \\
\hline Borda & $12,8 \pm 2,8 \mathrm{aA}$ & 7 & 5,4 \\
\hline Faixa 1 & $15,4 \pm 1,3 \mathrm{aA}$ & 8 & 5,9 \\
\hline Faixa 2 & $14,9 \pm 1,7 \mathrm{aA}$ & 7 & 5,1 \\
\hline \multirow[t]{2}{*}{ Interior } & $11,4 \pm 1,6 \mathrm{aAB}$ & 5 & 3,8 \\
\hline & \multicolumn{3}{|c|}{ Outono } \\
\hline Borda & $7,4 \pm 0,7 \mathrm{aA}$ & 6 & 5,8 \\
\hline Faixa 1 & $9,0 \pm 1,4 \mathrm{aB}$ & 6 & 5,2 \\
\hline Faixa 2 & $12,9 \pm 2,3 \mathrm{aAB}$ & 10 & 8,1 \\
\hline \multirow[t]{2}{*}{ Interior } & $11,9 \pm 3,2 \mathrm{aAB}$ & 8 & 6,5 \\
\hline & \multicolumn{3}{|c|}{ Inverno } \\
\hline Borda & $10,0 \pm 2,3 \mathrm{bA}$ & 5 & 4,0 \\
\hline Faixa 1 & $8,1 \pm 0,2 \mathrm{bB}$ & 6 & 5,5 \\
\hline Faixa 2 & $8,1 \pm 0,7 \mathrm{bBC}$ & 6 & 5,5 \\
\hline Interior & $16,3 \pm 1,4 \mathrm{aA}$ & 4 & 2,5 \\
\hline
\end{tabular}

Em que: valores seguidos de letras minúsculas iguais, que comparam a densidade de esporos entre as faixas e as letras maiúsculas iguais, que comparam a densidade de esporos de cada uma das faixas entre as estações não diferem entre si pelo teste de Tukey a 5\% de significância.

Também foram verificadas variações na densidade de esporos quando se comparou cada uma das faixas entre as diferentes estações do ano. A faixa que representa a borda, embora posicionada na extremidade do fragmento, não apresentou variação na densidade de esporos no decorrer do ano, enquanto as faixas 1 e 2 apresentaram maior densidade no verão. No interior do fragmento, a menor densidade foi encontrada 
na primavera e a maior no inverno (Tabela 3). Nesse sentido, a correlação negativa significativa verificada entre precipitação e densidade de esporos da matriz do fragmento (Tabela 4) sugere uma diminuição da densidade de esporos com o aumento da incidência de chuvas.

Tendo em vista que a associação micorrízica responde a mudanças no regime de precipitação (MARTÍNEZ-GARCIA; MIRANDA; PUGNAIRE, 2012), como observado no interior do fragmento, é provável que outros fatores tenham interferido na manutenção e propagação dos FMA nas faixas mais próximas da borda, como, por exemplo, a variação sazonal da composição química e orgânica da serapilheira depositada sobre o solo. Tal composição interfere na capacidade das hifas de FMAs adquirirem nitrogênio e participarem da ciclagem de carbono decorrente da decomposição (BRAGHIROLLI et al., 2012; HERMAN et al., 2012). Entretanto, a correlação da densidade de esporos com a serapilheira não mostrou coeficientes significativos para o estoque total, havendo correlação apenas para as frações galhos e cascas nas faixas $1 \mathrm{e}$ 2 , respectivamente (Tabela 4), o que sugere maior influência destes componentes na definição da qualidade da serapilheira.

TABELA 4: Coeficiente de correlação de Pearson entre a densidade de esporos da comunidade de FMA nas faixas do fragmento florestal e as variáveis climáticas temperatura e precipitação e o estoque de serapilheira (total e das frações).

TABLE 4: Pearson correlation coefficient between the spore densities of arbuscular mycorrhizal fungal (AMF) communities present in the bands of the forest fragment and the climate variables of temperature and precipitation and accumulated litter (total and fractions).

\begin{tabular}{ccccc}
\hline \multirow{2}{*}{ Correlação de Pearson } & \multicolumn{4}{c}{ Densidade de esporos de FMAs } \\
\cline { 2 - 5 } & Borda & Faixa 1 & Faixa 2 & Interior \\
\hline Temperatura & 0,61 & 0,55 & 0,37 & $-0,44$ \\
Precipitação & $-0,42$ & $-0,63$ & $-0,66$ & $\mathbf{- 0 , 9 7}$ \\
Serapilheira total & $-0,1$ & 0,5 & $-0,09$ & $-0,42$ \\
Folhas & 0,58 & $-0,50$ & 0,59 & 0,70 \\
Galhos & $-0,41$ & $\mathbf{0 , 9 7}$ & $-0,12$ & $-0,60$ \\
Cascas & 0,52 & 0,33 & $\mathbf{- 0 , 9 6}$ & 0,02 \\
M. reprodutivo & 0,16 & $-0,57$ & $-0,60$ & 0,51 \\
M. amorfo & $-0,51$ & $-0,15$ & $-0,32$ & $-0,27$ \\
\hline
\end{tabular}

Em que: números destacados em negrito correspondem a correlações significativas a $10 \%$ de probabilidade de erro. M. reprodutivo $=$ material reprodutivo, $\mathrm{M}$. amorfo $=$ material amorfo

Foram identificadas 24 espécies de FMA no fragmento florestal, no qual o maior número de espécies pertence ao gênero Acaulospora (6), seguido dos gêneros Glomus (3), Racocetra (3), Dentiscutata (3), Gigaspora (2), Archaeospora, Ambispora, Cetraspora, Claroideoglomus, Diversispora e Funneliformis (uma espécie cada) (Tabela 5).

Em determinados ambientes, a dominância de gêneros de FMA pode ser resultante da interação de fatores do ambiente, como, por exemplo, as características físico-químicas do solo, morfofisiológica das plantas, a compatibilidade genética entre fungo e planta, a dispersão dos fungos e a extinção de algumas espécies do local (ZANGARO; MOREIRA, 2010).

O número de espécies e o índice de diversidade não demonstraram um efeito nítido de sazonalidade ou de borda (Tabela 3). O índice demonstrou um possível efeito de borda apenas na primavera, quando evidenciou maior diversidade na matriz $(11,2)$, porém, este comportamento não se repetiu nas demais estações. O número de espécies apresentou pequena variação entre as faixas nas diferentes épocas do ano, estando entre 4 e 10 (Tabela 3 ).

Algumas espécies foram identificadas exclusivamente em uma faixa do fragmento florestal e, ou estação do ano. Dentre as espécies encontradas, quatro apresentaram esporos apenas na primavera, das quais duas eram do gênero Acaulospora, quatro no verão e três no outono, sendo que nenhuma espécie esporulou exclusivamente no inverno. A estação do ano interfere no ciclo de vida e época de esporulação entre as espécies de FMA. Todavia, a não ocorrência de esporos não indica a ausência da espécie de FMA e 
sua colonização radicular no ambiente edáfico, uma vez que podem estar presentes no solo apenas na forma de hifas, raízes colonizadas e/ou células auxiliares (OEHL et al., 2009; SANTOS; CARRENHO, 2011).

TABELA 5: Espécies de FMAs nas diferentes estações do ano e faixas do fragmento florestal avaliado.

TABLE 5:Species of arbuscular mycorrhizal fungi (AMF) in different seasons and forest fragment tracks.

\begin{tabular}{|c|c|c|c|c|}
\hline Espécies de FMA & Borda & Faixa 1 & Faixa 2 & Interior \\
\hline Acaulospora colombiana & & & $\mathrm{V}$ & \\
\hline Acaulospora foveata & O I & V I & PO I & $\mathrm{PO}$ \\
\hline Acaulospora mellea & & $\mathrm{P}$ & & $\mathrm{P}$ \\
\hline Acaulospora rehmii & & & & $\mathrm{P}$ \\
\hline Acaulospora scrobiculata & $\mathrm{P}$ & & P V I & $\mathrm{P}$ \\
\hline Acaulospora sp. & & & $\mathrm{O}$ & \\
\hline Acaulospora tuberculata & P I & P I & P O I & P I \\
\hline Ambispora leptoticha & $\mathrm{PO}$ & $\mathrm{O}$ & & \\
\hline Archaeospora undulata & $\mathrm{P}$ & & & \\
\hline Cetraspora pellucida & & $\mathrm{V}$ & P V & \\
\hline Claroideoglomus lamellosum & V & & & \\
\hline Dentiscutata cerradensis & & & & $\mathrm{O}$ \\
\hline Dentiscutata heterogama & & & & $\mathrm{P}$ \\
\hline Dentiscutata scutata & & $\mathrm{OI}$ & $\mathrm{O}$ & $\mathrm{I}$ \\
\hline Entrophospora sp. & & & $\mathrm{O}$ & \\
\hline Funneliformis verruculosum & $\mathrm{VO}$ & $\mathrm{O}$ & $\mathrm{VO}$ & $\mathrm{VO}$ \\
\hline Gigaspora gigantea & $\mathrm{P}$ & $\mathrm{PV}$ & $\mathrm{P}$ & $\mathrm{P}$ \\
\hline Gigaspora sp. & PVO I & V O I & V O I & V O I \\
\hline Glomus clavisporum & & $\mathrm{V}$ & & \\
\hline Glomus glomerulatum & $\mathrm{V}$ & $\mathrm{V}$ & & $\mathrm{V} \mathrm{O}$ \\
\hline Glomus macrocarpum & PVO I & PO I & PVO I & PVO I \\
\hline Glomus tortuosum & P V I & V I & & \\
\hline Racocetra verrucosa & $\mathrm{VO}$ & $\mathrm{O}$ & $\mathrm{VO}$ & $\mathrm{VO}$ \\
\hline
\end{tabular}

Em que: $\mathrm{P}=$ primavera; $\mathrm{V}=$ verão; $\mathrm{O}$ = outono; $\mathrm{I}=$ inverno.

Com relação à ocorrência de espécies, foram verificadas ocorrências exclusivas no gradiente bordainterior do fragmento: duas espécies na borda (Archaeospora undulata e Claroideoglomus lamellosum), uma na faixa 1 (Glomus clavisporum), duas na faixa 2 (Acaulospora colombiana e Acaulospora sp.) e três na matriz do fragmento (Acaulospora rehmii, Dentiscutata cerradensis e Dentiscutata heterogama). A ocorrência de maior número de espécies no interior do fragmento deve estar relacionada ao seu microclima, que, por ser mais estável, cria condições para o estabelecimento de mais espécies.

\section{CONCLUSÕES}

O efeito de borda em fragmentos de Floresta Estacional Semidecidual pode alterar o estoque de serapilheira (total e da fração folhas), que apresenta uma redução de massa nos locais mais próximos da borda, e a comunidade de FMA, que apresenta ocorrência de espécies exclusivas no gradiente borda-interior do fragmento. Portanto, estes indicadores podem ser empregados para conhecer os impactos decorrentes da fragmentação de habitat e auxiliar na prevenção de perdas de funções do ecossistema, bem como na definição de ações de proteção contra perturbações antrópicas. 


\section{REFERÊNCIAS}

ALVARES, C. A. et al. Köppen's climate classification map for Brazil. Meteorologische Zeitschrift, Stuttgart, v. 22, n. 6, p. 711-728, 2013.

BARBOSA, V. A. et al. Fitomassa e Conteúdos de C e N da Serapilheira Acumulada de Plantios de Madeira Nova e Eucalipto no Sudoeste da Bahia. In: FERTBIO: Reunião Brasileira de Fertilidade do Solo e Nutrição de Plantas, 30.; Reunião Brasileira sobre Micorrizas, 14.; Simpósio Brasileiro de Microbiologia do Solo, 12.; Reunião Brasileira de Biologia do Solo, 9.; Simpósio sobre Selênio no Brasil, 1., Maceió. Anais... [s. 1.: s. n.], 2012. 1 CD.

BATISTA, M. A.; TIMMERS, J.; CUNHA, R. P. P. Os estados da Mata Atlântica: Bahia. In: CAMPANILI, M.; PROCHNOW (Org.). Mata Atlântica - uma rede pela floresta. Brasília: RMA, 2006. p. 129-141.

BRAGHIROLLI, F. L. et al. Fungos micorrízicos arbusculares na recuperação de florestas ciliares e fixação de carbono no solo. Revista Brasileira de Ciência do Solo, Viçosa, MG, v. 36, n.3, p. 733-743, mai./jun. 2012.

BRASIL. Ministério do Meio Ambiente. Serviço Florestal Brasileiro. Florestas do Brasil em resumo: dados de 2005 - 2010. Brasília: SFB, 2010. 156 p.

BROWN, S.; LUGO, A. L. Tropical secondary forests. Journal of Tropical Ecology, Winchelsea, v. 6, p. 1-32, 1990.

DINIZ, A. R. et al. Biomassa, estoques de carbono e de nutrientes em estádios sucessionais da Floresta Atlântica, RJ. Revista Brasileira de Ciencias Agrárias, Recife, v. 10, n. 3, p. 443-451, 2015.

EMBRAPA. Sistema brasileiro de classificação de solos. 3. ed. rev. ampl. Brasília: Embrapa, 2013. 353 p. EUROPEAN ENVIRONMENT AGENCY. Environmental indicators: typology and use in reporting. [s. 1.]: European environment Agency, 2003. 20 p.

FAHRIG, L. Effects of habitat fragmentation on biodiversity. Annual Review of Ecology, Evolution and Systematics, Palo Alto, v. 34, p. 487-515, ago. 2003.

FERREIRA JÚNIOR, W. G.; SCHAEFER, C. E. G. R.; SILVA, A. F. Uma visão pedogeomorfológica sobre as formações florestais da Mata Atlântica. In: MARTINS, S. V. (Ed.). Ecologia de florestas tropicais do Brasil. 2. ed. rev. e ampl. Viçosa, MG: Ed. UFV, 2012. p. 141-174.

GARCIA, P. C. M. et al. Estoque e distribuição da serrapilheira em diferentes sistemas florestais. Revista Universidade Rural, Série Ciência da Vida, Seropédica, v. 25, n. 1, p. 12-17, 2005.

GASCON, C. WILLIAMSON, G. B.; FONSECA, G. A. B. Receding forest edges and vanishing reserves. Science, New York, v. 288, p. 1356-1358, may 2000.

GERDEMANN, J. W.; NICOLSON, T. H. Spores of mycorrhizal endogone species extracted from soil by wet-sieving and decanting. Transactions of British Mycological Society, Cambridge, v. 46, p. 235-244, 1963.

GRILLI, G.; URCELAY, C.; GALETTO, L. Forest fragment size and nutrient availability: complex responses of mycorrhizal fungi in native-exotic host. Plant Ecology, Dordrecht, v. 213, p. 155-165, sep. 2012.

HARPER, K. A. et al. Edge influence on forest structure and composition in fragmented landscapes. Conservation Biology, San Francisco, v. 19, n. 3, p. 768-782, jun. 2005.

HEGER, T. J.; IMFELD, G.; MITCHELL, E. A. D. Special issue on "bioindication in soil ecosystems": editorial note. European Journal of Soil Biology, Braunschweig, v. 9, p. 1-4, mar./apr. 2012.

HERMAN, D. J. et al. Interactions between an arbuscular mycorrhizal fungus and a soil microbial community mediating litter decomposition. FEMS Microbiology Ecology, Oxford, v. 80, p. 236-247, apr. 2012.

IBGE. Manual técnico da vegetação brasileira. 2. ed. rev. e ampl. Brasília: IBGE, 2012. 271 p.

JENKINS, W. R. A rapid centrifugal-flotation technique for separating nematodes from soil. Plant Disease Report, Beltsville, v. 28, p. 692, 1964.

KLEINPAUL, I. S. et al. Suficiência amostral para coletas de serapilheira acumulada sobre o solo em Pinus elliottii Engelm, Eucalyptus sp. e Floresta Estacional Decidual. Revista Árvore, Viçosa, MG, v. 29, n. 6, p. 965-972, nov./dez. 2005.

LAURANCE, W. F. et al. Ecosystem decay of Amazonian forest fragments: a 22-year investigation. Conservation Biology, San Francisco, v. 16, p. 605-618, may. 2002. 
LAURANCE, W. F. et al. The fate of Amazonian fores fragments: a 32-year investigation. Biologial Conservation, Boston, v. 144, p. 56-67, jan. 2011.

LAURANCE, W. F. et al. Rain forest fragmentation and the dynamics of Amazonian tree communities. Ecology, New York, v. 79, p. 2032-2040, sep. 1998.

LAURANCE, W. F. et al. Rain-forest fragmentation and the phenology of Amazonian tree Communities. Journal of Tropical Ecology, Winchelsea, v. 19, p. 343-347, may 2003.

LEAKE, J. R. et al. Networks of power and influence: the role of mycorrhizal mycelium in controlling plant communities and agroecosystem functioning. Canadian Journal of Botany, Ontario, v. 82, n. 8, p. 1016-1045, 2004.

MACHADO, M. R.; PIÑA-RODRIGUES, F. C. M.; PEREIRA, M. G. Produção de serrapilheira como indicador de recuperação em plantio adensado de revegetação. Revista Árvore, Viçosa, MG, v. 32, n. 1, p. 143-151, jan./fev. 2008

MARTÍNEZ-GARCÍA, L. B.; MIRANDA, J. D.; PUGNAIRE, F. I. Impacts of changing rainfall patterns on mycorrhizal status of a shrub from arid environments. European Journal of Soil Biology, Braunschweig, v. 50, p. 64-67, may/jun. 2012.

NÚCLEO MATA ATLÂNTICA. A Mata Atlântica na Bahia. [2013]. Disponível em: <http://www.ceama. mp.ba.gov.br/mata-atlantica.html?id=1353>. Acesso em: 1 abr. 2013.

NUNES, F. P.; PINTO, M. T. C. Decomposição do folhedo em reflorestamento ciliar na bacia hidrográfica do rio São Francisco, Minas Gerais. Cerne, Lavras v. 18, n. 3, p. 423-431, 2012.

OEHL, F. et al. Distinct sporulation dynamics of arbuscular mycorrhizal fungal communities from different agroecosystems in long-term microcosms. Agriculture, Ecosystems \& Environment, Amsterdam, v. 134, p. 257-268, dec. 2009.

PAGANO, M. C. et al. Plant-type dependente changes in arbuscular mycorrhizal communities as soil quality indicator in semi-arid Brazil. Ecological Indicators, Amsterdam, v. 11, p. 643-650, mar. 2011.

PENG, S.; GUO, T.; LIU, G. The effects of arbuscular mycorrhizal hyphal networks on soil aggregations of purple soil in southwest China. Soil Biology \& Biochemistry, Elmsford, p. 57, n. 411-417, feb. 2013. PRIMACK, R. B.; RODRIGUES, E. Biologia da conservação. Londrina: Ed. Rodrigues, 2001. 327 p.

RIBEIRO, M. C. et al. The Brazilian Atlantic Forest: how much is left, and how is the remaining forest distributed? Implications for conservation. Biological Conservation, Boston, v. 142, p. 1141-1153, jun. 2009.

RIGUEIRA, D. M. G. et al. Influência da distância da borda e do adensamento foliar sobre a abundância de plantas pioneiras em um fragmento de floresta tropical submontana na Estação Ecológica de Wenceslau Guimarães (Bahia, Brasil). Acta Botânica Brasilica, Feira de Santana, v. 26, p. 1, p. 197-202, jan./ mar. 2012.

RIUTTA, T. et al. Experimental evidence for the interacting effects of forest edge, moisture and soil macrofauna on leaf litter decomposition. Soil Biology \& Biochemistry, Elmsford, v. 49, p. 124-131, jun. 2012.

SANTOS, F. E. F.; CARRENHO, R. Diversidade de fungos micorrízicosarbusculares em remanescente florestal impactado (Parque Cinquentenário - Maringá, Paraná, Brasil). Acta Botanica Brasilica, Feira de Santana, v. 25, n. 2, p. 508-516, abr./jun. 2011.

SCARIOT, A. Vegetação e flora. In: RAMBALDI, D. M.; OLIVEIRA, D. A. S. (Org.). Fragmentação de ecossistemas: causas, efeitos sobre a biodiversidade e recomendações de políticas públicas. Brasília: MMA; SBF, 2003. p. 103-124.

SCHENCK, N. C.; PEREZ, Y. A manual of identification of vesicular-arbuscular mycorrhizal fungi. 2. ed. Florida: Gainesville, 1988. 241 p.

SOARES FILHO, A. O. Fitogeografia e estrutura das florestas estacionais deciduais no Brasil. 2012. 346 f. Tese (Doutorado) - Universidade Estadual de Feira de Santana, Feira de Santana, 2012.

SPERANDIO, H. V. et al. Emprego da serapilheira acumulada na avaliação de sistemas de restauração florestal em Alegre-ES. Floresta e Ambiente, Seropédica, v. 19, n. 4, p. 460-467, out./dez. 2012

SMITH, S. E.; READ, D. J. Mycorrhizal symbiosis. London: Academic Press, 2008. 787 p.

STENBERG, B. Monitoring soil quality of arable land: microbiological indicators. Soil and Plant Science, Copenhagen, v. 49, p. 1-24, aug. 1999. 
STÜRMER, S. L.; SIQUEIRA, J. O. Species richness and spore abundance of arbuscular mycorrhizal fungi across distinct land uses in Western Brazilian Amazon. Mycorrhiza, Adelaide, v. 21, n. 4, p. 255-267, may 2010.

VIERA, M. et al. Nutrientes na serapilheira em um fragmento de floresta estacional decidual, Itaara, RS. Ciência Florestal, Santa Maria, v. 20, n. 4, p. 611-619, out./dez. 2010.

VITOUSEK, P. M.; SANFORD JÙNIOR, R. L. Nutrient cycling in moist tropical forest. Annual Review of Ecology and Systematics, Palo Alto, v. 17, p. 137-167, 1986.

VOGEL, H. L. M. et al. Efeito de borda no estoque de serapilheira e nutrientes em um fragmento de floresta nativa no Bioma Pampa-RS. Ecologia e Nutrição Florestal, Santa Maria, v. 1, n. 1, p. 46-54, jan/abr. 2013. ZANGARO, W.; MOREIRA, M. Micorrizas arbusculares nos biomas Floresta Atlântica e Floresta de Araucária. In: SIQUEIRA, J. O. et al. (Ed.). Micorrizas: 30 anos de pesquisas no Brasil. Lavras: UFLA, 2010. p. 279-310. 